\title{
Flexible Maintenance Scheduling of Generation System by Multi- Probabilistic Reliability Criterion in Korea Power System
}

\author{
Jeongje Park*, Jaeseok Choi ${ }^{\dagger}$, Ungki Baek**, Junmin Cha*** and Kwang Y. Lee****

\begin{abstract}
A new technique using a search method which is based on fuzzy multi-criteria function is proposed for GMS(generator maintenance scheduling) in order to consider multi-objective function. Not only minimization of probabilistic production cost but also maximization of system reliability level are considered for fuzzy multi-criteria function. To obtain an optimal solution for generator maintenance scheduling under fuzzy environment, fuzzy multi-criteria relaxation method(fuzzy search method) is used. The practicality and effectiveness of the proposed approach are demonstrated by simulation studies for a real size power system model in Korea in 2010.
\end{abstract}

Keywords: Generator maintenance scheduling, Fuzzy set theory, Fuzzy multi-criteria functions

\section{Introduction}

The primary function of an electric power system is to provide electrical energy to its customers as economically as possible and with an acceptable degree of continuity and reliability.

It is, however, impossible to predict load exactly. A proper supply reliability all the time is not easy. Practically, the development of industrial utility has made the size of generation system huge and the system structure has been very complex. Therefore, problems about operation and planning of generation system are complicated[1]-[2].

Generator maintenance scheduling problem is an important planning problem that affects both economy and reliability for operation and planning of generating systems. Optimal maintenance scheduling is able, not only to raise supply and reserve rate, but also to postpone the period of construction cost of the generators, production cost and maintenance scheduling cost. The important point of generator maintenance scheduling is how to choose the objective function, and until now, maintenance scheduling problem has been built using the following objective functions [3]-[4] ;

(1) Generation Cost Minimization Method

(2) Levelized Risk Method

(3) Levelized Reserve Method

(4) Multi-criteria Functions Method

Additionally, recently methods for considering the capacity of transmission line have been proposed in [5]-[6]. In the present study, the method of maintenance scheduling

$\dagger$ Corresponding Author: Dept. of Electrical Engineering, ERI, Gyeongsang National University, Korea, (jschoi@gnu.ac.kr)

* Dept. of Electrical Engineering, Gyeongsang National Univerity, Korea. (pakjan@hanmail.net)

** Korea Western Power Co., Korea. (hot2217@westernpower.co.kr)

*** Daejin University, Korea. (chamin@daejin.ac.kr)

**** Baylor University, USA. (Kwang_Y_Lee@baylor.edu)

Received : May 25, 2009; Accepted : October 6, 2009 by fuzzy search method has been developed. It is expected that more flexible solution can be obtained because fuzzy set theory that can reflect the subjective decision of decision-maker has been used in this study[7]-[11]. An alternative method for flexible GMS using various kinds of objective functions is proposed in this paper. The effectiveness of the proposed approach are demonstrated by simulation studies of a real size power system model.

\section{Fuzzy Search Method}

The fuzzy decision set $\mathrm{D}$ resulting from fuzzy sets of $\mathrm{q}$ fuzzy goals, $G_{1}, G_{2}, \cdots, G_{q}$ and fuzzy sets of $p$ fuzzy constraints, $\mathrm{C}_{1}, \mathrm{C}_{2}, \cdots, \mathrm{C}_{\mathrm{p}}$ is as intersection defined as follows.

$$
D=\left(\bigcap_{i=1}^{p} G_{i}\right) \cap\left(\bigcap_{j=1}^{q} C_{i}\right)
$$

The membership function $\mu_{\mathrm{D}}$ resulting from the membership function of fuzzy sets of goals and constraints is defined as follows [12]:

$$
\mu_{D}(x)=\min \left[\min _{i=1 \sim p} \mu_{G} \cdot \min _{j=1 \sim q} \mu_{C}\right]
$$

where, min is an abbreviation of minimum.

If the fuzzy mathematical programming problem consists of finding maximum point of the membership function of the fuzzy decision set $\mathrm{D}$, the optimal solution can be obtained as:

$$
\mu_{D}\left(\mathrm{X}^{*}\right)=\max \mu_{D}(\mathrm{X})
$$

Where, $\mathbf{x}^{*}$ is the optimal decision solution, and $\max$ is an abbreviation of maximum.

The vector form in Eq.(3) can be rewritten as : 


$$
\mu_{D}\left(X_{1}^{*}, X_{2}^{*}, \ldots, X_{N}^{*}\right)=\max _{X_{1}, \ldots, X_{N}} \mu_{D}\left(X_{1}, X_{2}, \ldots, X_{N}\right)
$$

In order to solve this problem by using the fuzzy search method, the principle of optimality can be applied after Eq.(4) can be reformulated as:

$$
\begin{aligned}
\mu_{D}\left(X_{1}^{*}, X_{2}^{*}, \ldots, X_{N}^{*}\right)= & \max _{X_{2}, \ldots, X_{N}}\left[\operatorname { m a x } _ { X _ { 1 } } \left\{\min \mu_{D}\left(X_{1}\right), \mu_{F 2}\left(X_{2}\right)\right.\right. \\
& \left.\left.\left., \ldots, \mu_{F N-1}\left(X_{N-1}\right), \mu_{F N}\left(X_{N}\right)\right)\right\}\right] \\
& =\max _{X_{2}, \ldots, X_{N}}\left[\operatorname { m i n } \left\{\mu_{D}\left(X_{1}^{*}\right), \mu_{F 2}\left(X_{2}\right), \ldots,\right.\right. \\
& \left.\mu_{F N-1}\left(X_{N-1}\right), \mu_{F N}\left(X_{N}\right)\right]
\end{aligned}
$$

where, $\mathrm{X}$ : decision variable, $\mathrm{F}=\mathrm{G}+\mathrm{C}$ (algebraic sum of fuzzy sets)

Eq.(5) can be rewritten as:

$$
\begin{array}{r}
\mu_{D}\left(X_{1}^{*}, X_{2}^{*}, \ldots, X_{N}^{*}\right)=\max _{X_{n}, \ldots, X_{N}}[ \\
\min \left\{\mu_{D}\left(X_{1}^{*}, X_{2}^{*}, \ldots, X_{N-1}^{*}\right),\right. \\
\left.\left.\mu_{F n}\left(X_{n}\right), . ., \mu_{F N}\left(X_{N}\right)\right\}\right]
\end{array}
$$

And, we can also rewrite Eq.(6) as:

$$
\begin{array}{r}
\mu_{D}\left(X_{1}^{*}, X_{2}^{*}, \ldots, X_{n}^{*}\right)=\max _{X_{n}}\left[\operatorname { m i n } \left\{\mu_{D}\left(X_{1}^{*}, X_{2}^{*}, \ldots, X_{n-1}^{*}\right),\right.\right. \\
\left.\left.\mu_{F n}\left(X_{n}\right)\right\}\right] \\
\mu_{D}\left(S_{n}\right)=\max _{X_{n}}\left[\min \left\{\mu_{D}\left(S_{n-1}\right), \mu_{F n}\left(X_{n}\right)\right\}\right]
\end{array}
$$

where, $\mathrm{S}_{\mathrm{n}}=f\left(\mathrm{~S}_{\mathrm{n}-1}, \mathrm{X}_{\mathrm{n}-1}\right) \mathrm{n}=1,2, \ldots \ldots \mathrm{N}$

$\mathrm{S}$ : state variable

$f$ : state transition function

\section{The Formulation based on Fuzzy Search Method}

\subsection{The Formulation based on Fuzzy Search Method}

(1) Minimization of Probabilistic Production Cost F as:

$$
\begin{aligned}
& \text { Minimize } F\left\{E_{i n}, \Phi_{i}\left(U_{i n}\right)\right\} \\
& =\sum_{n=1}^{N T} \sum_{i=1 .}^{N G}\left\{A_{i} E_{i n}+B_{i} T \Phi_{i}\left(U_{i n}\right)\right\}^{[\$]}
\end{aligned}
$$

where, $A_{i}$ is one dimensional coefficient of fuel cost function[\$ $/ \mathrm{MWh}$, and $\mathrm{B}_{\mathrm{i}}$ is constant of fuel cost function[\$].

$$
E_{i n}=\left(1-q_{i}\right) T \int_{u i-1}^{u i} \Phi_{i n-1}(X) d X \quad[\mathrm{MWh}]
$$

$\mathrm{E}_{i n}$ : probabilistic generation energy of $\# i$ unit at \#n stage $\mathrm{T}:$ total period for study [hours]

$i$ : number of the economic order of generators $\mathrm{u}_{i}=\mathrm{C}_{1}+\mathrm{C}_{2}+\cdots+\mathrm{C}_{\mathrm{i}}[\mathrm{MW}]$
$\mathrm{C}_{i}$ : capacity of $\# i$ unit

$\mathrm{u}_{0}=0$

$\phi_{i n}$ : effective load duration curve

$\mathrm{q}_{i}$ : forced outage rate of $\# i$ unit

Given aspiration level of decision-maker for the probabilistic production cost Eq.(8) can be represented as fuzzy goal function form as :

$$
F\left\{E_{i n} F_{i}\left(U_{i n}\right)\right\} \leqq Z_{o t}
$$

where, $\mathrm{Z}_{01}$ : aspiration level of decision-maker for the production cost

\section{(2) Minimization of LOLE}

Minimizing of LOLE is defined;

$$
\text { Minimize } Z_{2}=L O L E=\Phi_{N G n}\left(U_{N G n}\right) \quad[\mathrm{pu}]
$$

And also, Eq.(11) can be to represented as fuzzy goal function form as:

$$
\mathrm{Z}_{2} \gtrsim \mathrm{Z}_{02}
$$

Where, $\mathrm{Z}_{02}$ : aspiration level of decision-maker for LOLE

\section{(3) Maximization of Minimum $S R R_{n}$}

Maximizing of minimum SRR(Supply Reserve Rate) in 52 weeks should be considered. It can be defined as:

$$
\text { Maximize } \mathrm{Z}_{3}=\text { minimum }\left(\mathrm{SRR}_{\mathrm{n}}\right) \quad[\%]
$$

Where, $\mathrm{SRR}_{\mathrm{n}}=\left(\mathrm{IC}-\mathrm{MCAP}_{\mathrm{n}}-\mathrm{PD}_{\mathrm{n}}\right) \times 100 / \mathrm{PD}_{\mathrm{n}}$

IC: total installed capacity of generators [MW]

$\mathrm{MCAP}_{\mathrm{n}}$ : maintenance capacity at $\mathrm{n}$-th week [MW]

$\mathrm{PD}_{\mathrm{n}}$ : peak load at n-th week [MW]

\section{(4) Maximization of EIR}

Maximizing of EIR(Energy Index of Reliability) can be considered as:

$$
\text { Maximize } \mathrm{Z}_{4}=\mathrm{EIR} \text { [pu] }
$$

\section{Where, EIR=1-EENS/ESD}

EENS: expected energy not served [MWh]

ESD: expected energy for demand [MWh]

And also, Eq.(12) can be to represented as fuzzy goal function form as:

$$
Z_{4} \gtrsim Z_{04}
$$

where, $\mathrm{Z}_{04}$ : aspiration level of decision-maker for EDNS. 


\subsection{Constraints}

\section{(1) Boundary Conditions}

$$
\begin{aligned}
& \mathbf{X}(1)=\underline{\mathbf{0}} \\
& \mathbf{X}(T+1)=\operatorname{col}\left[M D_{1}, M D_{2}, M D_{3}, \ldots, M D_{N G}\right]^{T}
\end{aligned}
$$

Where, $\underline{\mathbf{0}}$ : zero vector

$\mathrm{MD}_{i}$ : time period asked for maintenance of $\# i$ unit

(2) Constraints for Maintenance Possible Time Period

$$
U_{i}(t)=\left\{\begin{array}{rr}
0 & t<M S_{i} \text { or } t>M F_{i}+M D_{i} \\
1 & M S_{i} \leq t \leq M F_{i}+M D_{i}
\end{array}\right\}
$$

where, $\mathrm{MS}_{i}$ : starting time for maintenance of first possible maintenance time period of $\# i$ unit

$\mathrm{MF}_{i}$ : starting time for maintenance of last possible maintenance time period of $\# i$ unit

(3) Constraints for Maintenance Possible Time Period

$$
\sum_{i \in P_{k}} U_{i}(t) \leq 1
$$

where, $\mathrm{P}_{\mathrm{k}}$ : set of generators at \#k power plant

\section{(4) Constraint of Maintenance Equipments}

$$
\sum_{i=1}^{N G} U_{i}(t) \cdot M_{k l i} \leq M A_{k}(t)
$$

where, $\mathrm{k}$ : the number of the kinds of maintenance equipment $(k=1,2, \ldots, \mathrm{K})$

1: number of maintenance scheduled time of $\# i$ unit

$\mathrm{MA}_{k}(\mathrm{t})$ : amount of \#k maintenance equipment available within \#t stage

$\mathrm{M}_{k l i}$ : amount of $\# k$ maintenance equipment within $\# l$ maintenance time period of $\# i$ unit

\section{Establishment of Membership Function}

(1) Membership Function of Fuzzy Set for The Production Cost is Defined as:

$$
\mu_{c}\{X(t-1), u(t)\}=\left\{\begin{array}{cc}
1 & : \Delta C(\cdot) \leq 0 \\
e^{-W_{C} \Delta \varepsilon\{X(t-1), u(t)\}} & : \Delta C(\cdot)\rangle 0
\end{array}\right\}
$$

where, $\mu_{c}(\cdot)$ : membership function of fuzzy set for production cost

$\Delta \mathrm{C}(\cdot)=\{\mathrm{F}(\mathrm{X}(\mathrm{t})-\mathrm{Casp}(\mathrm{t})\} / \operatorname{Casp}(\mathrm{t})$

Casp $(\mathrm{t})$ : aspiration level for production cost at \#t stage

$\mathrm{W}_{\mathrm{c}}$ : weighting factor of the membership function for production cost
(2) Membership Function of Fuzzy Set for the Reliability LOLE is Defined as:

$$
\mu_{\text {LOLE }}\{X(t-1), u(t)\}=\left\{\begin{array}{cc}
1 & : \Delta R(\cdot) \leq 0 \\
e^{-W_{\text {LOLE }} \Delta R\{X(t-1), u(t)\}} & : \Delta R(\cdot)\rangle 0
\end{array}\right\}
$$

where, $\mu_{\mathrm{LOLE}}(\cdot)$ : membership function of fuzzy sets for reliability LOLE

$$
\Delta \mathrm{R}(\cdot)=\{\mathrm{RES}(\mathrm{X}(\mathrm{t})-\mathrm{REQ}(\mathrm{t})\} / \mathrm{REQ}(\mathrm{t})
$$

$\mathrm{REQ}(\mathrm{t})$ : aspiration level for reliability at \#t stage

$\mathrm{W}_{\text {LOLE }}$ : weighting factor of the membership function for reliability LOLE

(3) Membership Functions of Fuzzy Set for the Reliability SRR is Defined as:

$$
\mu_{S R R}\{X(t-1), u(t)\}=\left\{\begin{array}{cc}
1 & : \Delta R(\cdot) \leq 0 \\
e^{W_{S R R} \Delta R\{X(t-1), u(t)\}} & : \Delta R(\cdot)\rangle 0
\end{array}\right\}
$$

where, $\mu_{\mathrm{SRR}}(\cdot)$ : membership function of fuzzy sets for reliability SRR

$$
\begin{aligned}
& \Delta \mathrm{R}(\cdot)=\{\operatorname{RES}(\mathrm{X}(\mathrm{t})-\mathrm{REQ}(\mathrm{t})\} / \mathrm{REQ}(\mathrm{t}) \\
& \mathrm{REQ}(\mathrm{t}): \text { aspiration level for reliability SRR at \#t }
\end{aligned}
$$
stage

$\mathrm{W}_{\text {SRR }}$ : weighting factor of the membership function for reliability SRR

(4) Membership Function of Fuzzy Set for the Reliability EIR is Defined as:

$$
\mu_{E I R}\{X(t-1), u(t)\}=\left\{\begin{array}{cc}
1 & : \Delta R(\cdot) \leq 0 \\
e^{-W_{E I R} \Delta A\{X(t-1), u(t)\}} & : \Delta R(\cdot)\rangle 0
\end{array}\right\}
$$

where, $\mu_{\mathrm{EIR}}(\cdot)$ : membership function of fuzzy sets for air pollution fuzzy set

$\Delta \mathrm{R}(\cdot)=\{\mathrm{RES}(\mathrm{X}(\mathrm{t})-\mathrm{REQ}(\mathrm{t})\} / \mathrm{REQ}(\mathrm{t})$

$\mathrm{REQ}(\mathrm{t})$ : aspiration level for reliability EIR at \#t stage

$\mathrm{W}_{\mathrm{EIR}}$ : weighting factor of the membership function for reliability EIR

\section{Solution Procedure by the Fuzzy Search Method}

Fuzzy decision set D applied to the Eq.(1) can be formulated as:

$$
D=C \cap R 1 \cap R 2 \bigcap R 3
$$

where, C: fuzzy set for production cost

R1: fuzzy set for reliability SRR

R2: fuzzy set for reliability LOLE

R3: fuzzy set for reliability EIR

Therefore, using Eq.(7), we can obtain: 


$$
\mu_{D}(X(t))=\max \left[\min \left\{\mu_{C}(\cdot), \mu_{R 1}(\cdot), \mu_{R 2}(\cdot), \mu_{A}(\cdot), \mu_{D}(\mathrm{X}(\mathrm{t}-1))\right\}\right.
$$

where, $\mathrm{X}(\mathrm{t})=\mathrm{X}(\mathrm{t}-1)+\mathrm{u}(\mathrm{t})$

$\mu_{\mathrm{D}}(\mathrm{X}(0))=1.0$

$\mu_{\mathrm{D}}(\cdot)$ :membership function of fuzzy set for decision function

\section{Case Study}

\subsection{Input Data}

The proposed method was applied to the Korea power system on 2010 and probability production cost was calculated by the cumulant method. Fig. 1 represents year load curve pattern which has weekly load peaks on 2010 in the KEPCO system. The year peak load in this year is forecasted as $62,852 \mathrm{MW}$. Table 1 shows generation system in 2010.

Aspiration levels and weighting factors for probability production cost, deterministic and probabilistic reliability indices shown in Table 1 has been used for this case study.

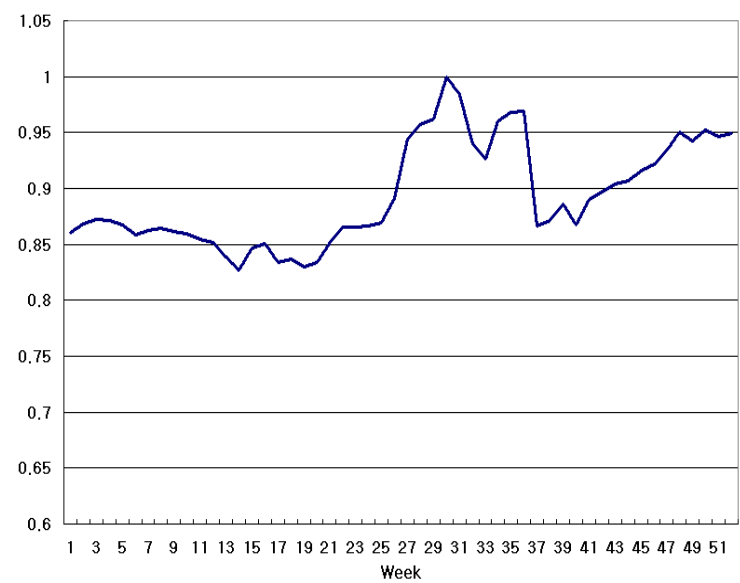

Fig. 1. Year load curve pattern with weekly load peaks.

Table 1. Input data (aspiration level \& weighting factor)

\begin{tabular}{c|c|c}
\hline & aspiration & weighting factor \\
\hline $\mathrm{Z}_{01}$ & $25,400,000\left[10^{6} \mathrm{Won}\right]$ & 10.0 \\
\hline $\mathrm{Z}_{02}$ & $10.0[\% /$ year $]$ & 5.0 \\
\hline $\mathrm{Z}_{03}$ & $5.5[$ days/year $]$ & 5.0 \\
\hline $\mathrm{Z}_{04}$ & $0.99900[\mathrm{pu}]$ & 5.0 \\
\hline
\end{tabular}

\subsection{Output Data}

The results are in appendix. Fig. 2 shows convergence of the membership value of objective function according to iterations. The objective function which indicates the satisfaction degree of decision-maker converges over 0.9 from fourth iteration. The convergence maximum $\mu_{\max }{ }^{*}$ is 0.952 at fourteen iterations. Fig. 6 is a representation of maintenance of power and supply reserve rates for each week.

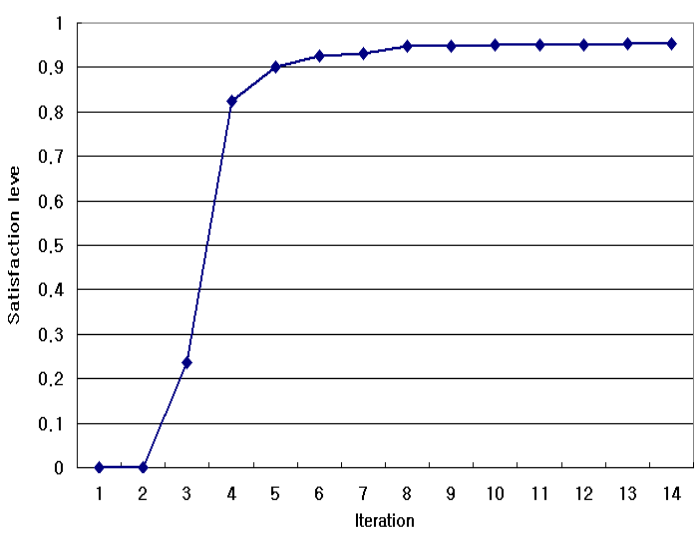

Fig. 2. Convergence of the objective function $\left(\mu_{\max }\right)$.

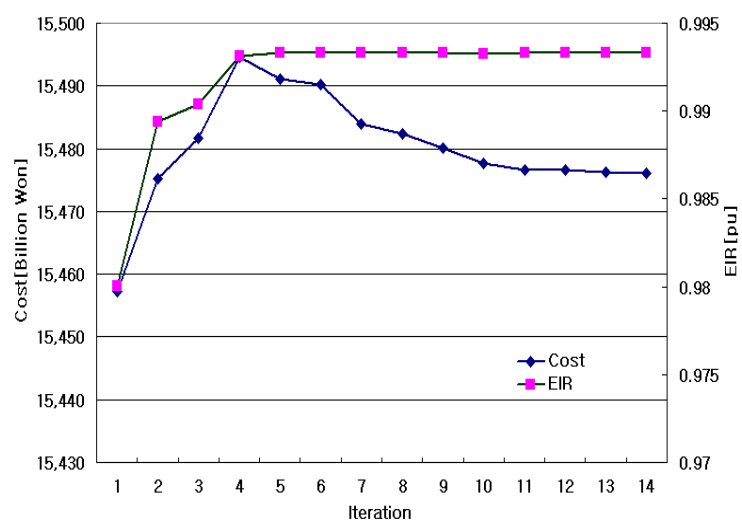

Fig. 3. Cost and EIR.

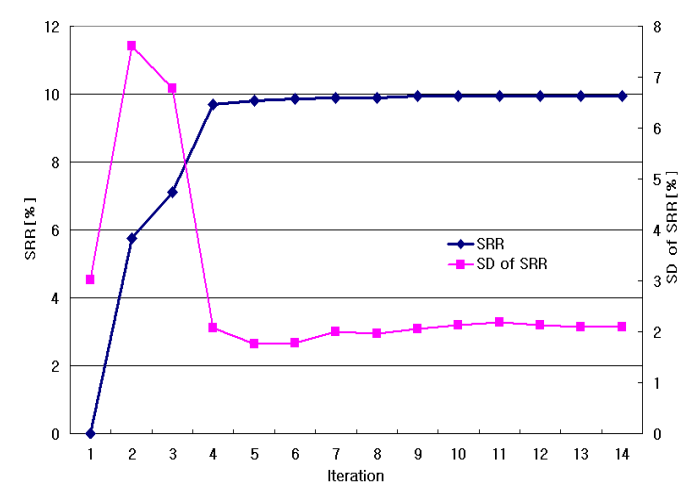

Fig. 4. SRR and standard deviation.

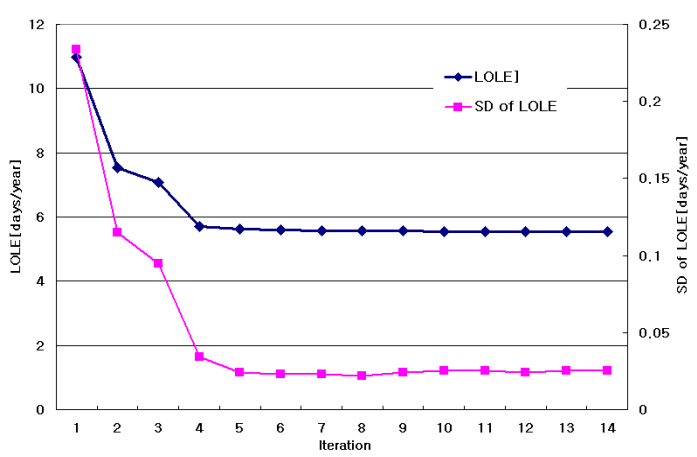

Fig. 5. LOLE and standard deviation. 


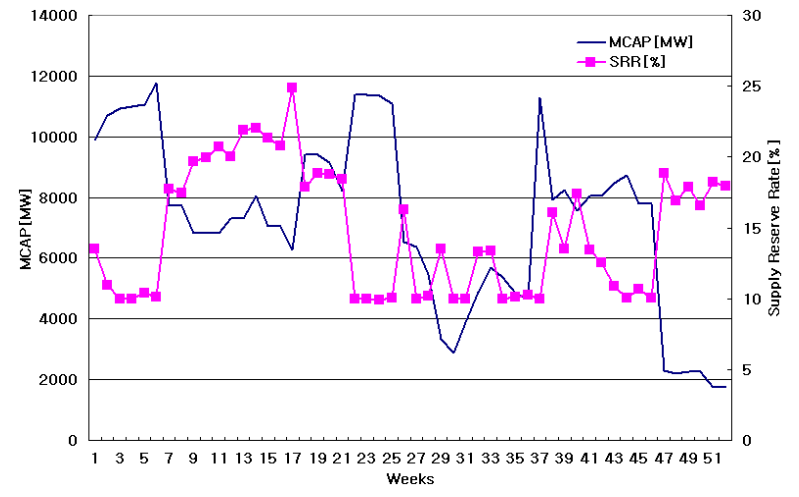

Fig. 6. Maintenance of powers and supply reserve rates.

\subsection{Parametric Analysis}

\section{Case study I}

Table 3 shows a parametric analysis result according to changing of aspiration level of SRR. As the aspiration level of the SRR is higher (stricter), the propose method asks for sacrifices of other objective values, cost, LOLE and EIR in order to avoid sharp decreasing of the satisfaction level as possible as. As it is, the cost and LOLE are asked for their increasing a little bit. EIR is asked for its decreasing a little bit. Therefore, the sharp decreasing of satisfaction level due to higher aspiration level of the SRR may be avoided overall. Eventually, more flexible solution is obtained using proposed method

Table 2. Aspiration Levels and Weighting Factors of Membership Functions for Cases 1, 2 and 3

\begin{tabular}{c|c|c|c|c}
\hline & Case 1 & Case 2 & Case 3 & $\begin{array}{c}\text { Weighting } \\
\text { Factor }\end{array}$ \\
\hline $\begin{array}{c}\text { Cost } \\
\text { [106 Won }]\end{array}$ & $15,400,000$ & $15,400,000$ & $15,400,000$ & 10 \\
\hline SRR[\%] & 10.0 & 12.0 & 14.0 & 5 \\
\hline $\begin{array}{c}\text { LOLE } \\
\text { [days/year] }\end{array}$ & 5.5 & 5.5 & 5.5 & 5 \\
\hline EIR[pu] & 0.99900 & 0.99900 & 0.99900 & 5 \\
\hline
\end{tabular}

Table 3. Results According to Changing of Aspiration Level of SRR(Supply Reserve Rate)

\begin{tabular}{c|c|c|c|c}
\hline & Case 1 & Case 2 & Case 3 & Remarks \\
\hline $\begin{array}{c}\text { Cost } \\
{\left[10^{6} \mathrm{Won}\right]}\end{array}$ & $15,476,098$ & $15,497,311$ & $15,513,394$ & Increasing \\
\hline SRR[\%] & 9.957 & 11.707 & 13.218 & Increasing \\
\hline $\begin{array}{c}\text { LOLE } \\
\text { days/year] }\end{array}$ & 5.554 & 5.632 & 5.804 & Increasing \\
\hline EIR[pu] & 0.99328 & 0.99332 & 0.99319 & Decreasing \\
\hline $\begin{array}{c}\text { Satisfaction } \\
\text { Level }\end{array}$ & 0.952 & 0.885 & 0.756 & Decreasing \\
\hline
\end{tabular}

\section{Case study II}

Table 5 shows a parametric analysis result according to changing of aspiration level of production cost. As the aspiration level of the cost is lower(stricter), the propose
Table 4. Aspiration Levels and Weighting Factors of Membership Functions for Cases 5 and 6

\begin{tabular}{c|c|c|c|c}
\hline & Case 1 & Case 5 & Case 6 & $\mathrm{W}_{\mathrm{R}}$ \\
\hline $\begin{array}{c}\text { Cost } \\
{\left[10^{6} \text { Won }\right]}\end{array}$ & $15,400,000$ & $15,200,000$ & $15,000,000$ & 10 \\
\hline $\begin{array}{c}\text { SRR } \\
{[\%]}\end{array}$ & 10.0 & 10.0 & 10.0 & 5 \\
\hline $\begin{array}{c}\text { LOLE } \\
\text { days/year }]\end{array}$ & 5.5 & 5.5 & 5.5 & 5 \\
\hline $\begin{array}{c}\text { EIR } \\
{[\mathrm{pu}]}\end{array}$ & 0.99900 & 0.99900 & 0.99900 & 5 \\
\hline
\end{tabular}

Table 5. Results According to Changing of Aspiration Level of Production Cost

\begin{tabular}{c|c|c|c|c}
\hline & Case 1 & Case 5 & Case 6 & Remarks \\
\hline $\begin{array}{c}\text { Cost } \\
{\left[10^{6} \text { Won }\right]}\end{array}$ & $15,476,098$ & $15,472,839$ & $15,468,876$ & Decreasing \\
\hline SRR[\%] & 9.957 & 9.665 & 9.397 & Decreasing \\
\hline $\begin{array}{c}\text { LOLE } \\
\text { days/year] }\end{array}$ & 5.554 & 5.697 & 5.844 & Increasing \\
\hline $\begin{array}{c}\text { EIR } \\
\text { [pu] }\end{array}$ & 0.99328 & 0.99298 & 0.99267 & Decreasing \\
\hline $\begin{array}{c}\text { Satisfaction } \\
\text { Level }\end{array}$ & 0.952 & 0.836 & 0.732 & Decreasing \\
\hline
\end{tabular}

method asks for sacrifices of other objective values, SRR, LOLE and EIR in order to avoid sharp decreasing of the satisfaction level as possible as. As it is, the LOLE is asked for their increasing a little bit. The SRR and EIR are asked for their decreasing a little bit. Therefore, the sharp decreasing of satisfaction level due to higher aspiration level of the cost may be avoided overall. Eventually, more flexible solution is obtained using proposed method.

\section{Conclusion}

A new technique using a search method which is based on fuzzy multi-criteria function is proposed for the GMS problem in order to consider multi-objective function. Not only minimization of probabilistic production cost but also various kinds of system reliability indices levels are considered simultaneously for fuzzy multi-criteria function. To obtain an optimal flexible solution for generator maintenance scheduling under fuzzy environment, fuzzy multicriteria relaxation method (fuzzy search method) is used.

When an aspiration level is stricter, the propose method suggests another optimal solution that avoids to decrease satisfaction level(objective function) sharply even if other objective values are asked for their sacrifices a little bit. It shows, eventually, that more flexible solution is obtained using proposed method. The practicability and effectiveness of the proposed of method are demonstrated by simulation results on real size power system model in Korea in 2010.

\section{Acknowledgements}

This work has been supported by KESRI(09309), which 
is funded by Korea Western Power Co. The support of the Advanced Power Network Reliability Research Center (APRRC) is acknowledged.

\section{Appendix}

Table 1. Generation system in Korea in 2010

\begin{tabular}{|c|c|c|c|c|c|c|c|c|c|}
\hline $\mathrm{NG}$ & Name & ID & $\begin{array}{c}\text { Cap. } \\
\text { [MW] }\end{array}$ & FOR & $\begin{array}{l}\mathrm{A}[\mathrm{Gcal} / \\
\left.\mathrm{MW}^{2} \mathrm{~h}\right]\end{array}$ & $\begin{array}{l}\text { B [Gcal/ } \\
\text { MWh] }\end{array}$ & $\mathrm{C}[\mathrm{Gcal} / \mathrm{hr}]$ & $\begin{array}{c}\text { Fuel cost } \\
{\left[10^{3} \mathrm{won} / \mathrm{Gcal}\right]}\end{array}$ & $\begin{array}{c}\text { Duration of } \\
\text { Maint. [weeks] }\end{array}$ \\
\hline 1 & WLSN & 2 & 700 & \begin{tabular}{|l|l|}
0.106 \\
\end{tabular} & 0 & 1.991 & 389.736 & 0.944 & 8 \\
\hline 2 & WLSN & 1 & 679 & \begin{tabular}{|l|l|}
0.106 \\
\end{tabular} & 0 & 2.002 & 389.736 & 0.946 & 8 \\
\hline 3 & WLSN & 3 & 700 & \begin{tabular}{|l|l|}
0.106 \\
\end{tabular} & 0 & 2.019 & 389.736 & 1.018 & 8 \\
\hline 4 & WLSN & 4 & 700 & \begin{tabular}{|l|l|}
0.106 \\
\end{tabular} & 0 & 2.019 & 389.736 & 1.019 & 8 \\
\hline 5 & ULJN & 1 & 950 & \begin{tabular}{|l|l|} 
\\
\end{tabular} & 0 & 1.958 & 348.674 & 1.233 & 8 \\
\hline 6 & ULJN & 2 & 950 & \begin{tabular}{|l|}
0.096 \\
\end{tabular} & 0 & 1.955 & 348.674 & 1.27 & 8 \\
\hline 7 & ULJN & 3 & 1000 & \begin{tabular}{|l|l|}
0.096 \\
\end{tabular} & 0 & 1.996 & 348.674 & 1.254 & 8 \\
\hline 8 & ULJN & 4 & 1000 & \begin{tabular}{|l|}
0.096 \\
\end{tabular} & 0 & 1.972 & 348.674 & 1.273 & 8 \\
\hline 9 & YNGN & 1 & 950 & \begin{tabular}{|l|}
0.096 \\
\end{tabular} & 0 & 2.029 & 275.783 & 1.28 & 8 \\
\hline 10 & YNGN & 2 & 950 & \begin{tabular}{|l|}
0.096 \\
\end{tabular} & 0 & 2.053 & 275.783 & 1.29 & 8 \\
\hline 11 & YNGN & 3 & 1000 & \begin{tabular}{|l|}
0.096 \\
\end{tabular} & 0 & 2.056 & 96.878 & 1.288 & 8 \\
\hline 12 & YNGN & 4 & 1000 & \begin{tabular}{|l|}
0.096 \\
\end{tabular} & 0 & 2.073 & 96.878 & 1.278 & 8 \\
\hline 13 & NGRI & 5 & 1000 & \begin{tabular}{|l|}
0.096 \\
\end{tabular} & 0 & 1.164 & 667.365 & 1.419 & 8 \\
\hline 14 & GORI & 1 & 587 & \begin{tabular}{|l|l|}
0.106 \\
\end{tabular} & 0 & 2.21 & 176.36 & 1.254 & 8 \\
\hline 15 & GORI & 2 & 650 & \begin{tabular}{|l|l|}
0.106 \\
\end{tabular} & 0 & 2.025 & 263.184 & 1.399 & 8 \\
\hline 16 & GORI & 3 & 950 & \begin{tabular}{|l|}
0.096 \\
\end{tabular} & 0 & 2.35 & 119.201 & 1.27 & 8 \\
\hline 17 & GORI & 4 & 950 & \begin{tabular}{|l|}
0.096 \\
\end{tabular} & 0 & 2.346 & 119.2 & 1.295 & 8 \\
\hline 18 & ULJN & 5 & 1000 & \begin{tabular}{|l|}
0.096 \\
\end{tabular} & 0.001 & 1.213 & 668.248 & 1.251 & 8 \\
\hline 19 & YNGN & 6 & 1000 & \begin{tabular}{|l|}
0.096 \\
\end{tabular} & 0.001 & 1.213 & 668.248 & 1.299 & 8 \\
\hline 20 & YNGN & 5 & 1000 & \begin{tabular}{|l|}
0.096 \\
\end{tabular} & 0.001 & 1.213 & 668.248 & 1.307 & 8 \\
\hline 21 & ULJN & 6 & 1000 & \begin{tabular}{|l|}
0.096 \\
\end{tabular} & 0.001 & 1.213 & 668.248 & 1.324 & 8 \\
\hline 22 & HADN & 5 & 500 & \begin{tabular}{|l|}
0.053 \\
\end{tabular} & 0 & 1.709 & 150.742 & 8.462 & 6 \\
\hline 23 & BORY & 6 & 500 & \begin{tabular}{|l|}
0.053 \\
\end{tabular} & 0 & 1.775 & 135.506 & 8.395 & 6 \\
\hline 24 & DNJN & 5 & 500 & \begin{tabular}{|l|}
0.053 \\
\end{tabular} & 0 & 1.671 & 152.67 & 8.983 & 6 \\
\hline 25 & DNJN & \begin{tabular}{|l|}
6 \\
\end{tabular} & 500 & \begin{tabular}{|l|}
0.053 \\
\end{tabular} & 0 & 1.711 & 143.251 & 8.983 & 6 \\
\hline 26 & HADN & 3 & 500 & \begin{tabular}{|l|}
0.053 \\
\end{tabular} & 0 & 1.817 & 129.229 & 8.462 & 6 \\
\hline 27 & BORY & 2 & 500 & \begin{tabular}{|l|}
0.053 \\
\end{tabular} & 0 & 1.844 & 138.866 & 8.395 & 6 \\
\hline 28 & BORY & 3 & 500 & \begin{tabular}{|l|}
0.053 \\
\end{tabular} & 0 & 1.851 & 122.508 & 8.395 & 0 \\
\hline 29 & DNJN & 1 & 500 & \begin{tabular}{|l|}
0.053 \\
\end{tabular} & 0 & 1.769 & 132.81 & 8.983 & 6 \\
\hline 30 & DNJN & 3 & 500 & \begin{tabular}{|l|}
0.053 \\
\end{tabular} & 0 & 1.781 & 130.192 & 8.983 & 6 \\
\hline 31 & DNJN & 7 & 500 & \begin{tabular}{|l|}
0.053 \\
\end{tabular} & 0 & 1.784 & 92.894 & 8.983 & 6 \\
\hline 32 & DNJN & 8 & 500 & \begin{tabular}{|l|}
0.053 \\
\end{tabular} & 0 & 1.784 & 92.894 & 8.983 & 6 \\
\hline 33 & DNJN & 4 & 500 & \begin{tabular}{|l|}
0.053 \\
\end{tabular} & 0 & 1.785 & 128.954 & 8.983 & 6 \\
\hline 34 & DNJN & 2 & 500 & \begin{tabular}{|l|}
0.053 \\
\end{tabular} & 0 & 1.795 & 128.351 & 8.983 & 6 \\
\hline 35 & TEAN & \begin{tabular}{|l|}
8 \\
\end{tabular} & 500 & \begin{tabular}{|l|}
0.053 \\
\end{tabular} & 0 & 1.698 & 103.771 & 9.602 & 6 \\
\hline 36 & TEAN & 2 & 500 & \begin{tabular}{|l|}
0.053 \\
\end{tabular} & 0 & 1.739 & 139.644 & 9.602 & 6 \\
\hline 37 & TEAN & 3 & 500 & \begin{tabular}{|l|}
0.053 \\
\end{tabular} & 0 & 1.739 & 139.644 & 9.602 & 6 \\
\hline 38 & TEAN & 4 & 500 & \begin{tabular}{|l|}
0.053 \\
\end{tabular} & 0 & 1.739 & 139.644 & 9.602 & 6 \\
\hline 39 & YNHN & 2 & 800 & \begin{tabular}{|l|}
0.042 \\
\end{tabular} & 0 & 1.85 & 168.042 & 9.157 & 8 \\
\hline 40 & SMCN & \begin{tabular}{|l|}
6 \\
\end{tabular} & 500 & \begin{tabular}{|l|}
0.053 \\
\end{tabular} & 0 & 1.87 & 111.914 & 9.063 & 6 \\
\hline 41 & SMCN & 5 & 500 & \begin{tabular}{|l|}
0.053 \\
\end{tabular} & 0 & 1.877 & 112.037 & 9.063 & 6 \\
\hline 42 & HADN & 6 & 500 & \begin{tabular}{|l|}
0.053 \\
\end{tabular} & 0 & 2.027 & 89.259 & 8.462 & 6 \\
\hline 43 & TEAN & 1 & 500 & \begin{tabular}{|l|}
0.053 \\
\end{tabular} & 0 & 1.789 & 130.662 & 9.602 & 6 \\
\hline 44 & YNHN & 1 & 800 & \begin{tabular}{|l|}
0.042 \\
\end{tabular} & 0 & 1.933 & 150.791 & 9.157 & 8 \\
\hline 45 & SMCN & 4 & 560 & \begin{tabular}{|l|}
0.053 \\
\end{tabular} & 0 & 1.956 & 118.25 & 9.063 & 6 \\
\hline 46 & TEAN & \begin{tabular}{|l|}
7 \\
\end{tabular} & 500 & \begin{tabular}{|l|}
0.053 \\
\end{tabular} & 0 & 1.848 & 107.291 & 9.602 & 6 \\
\hline 47 & TEAN & \begin{tabular}{|l|}
5 \\
\end{tabular} & 500 & \begin{tabular}{|l|}
0.053 \\
\end{tabular} & 0 & 1.86 & 120.76 & 9.602 & 6 \\
\hline 48 & TEAN & 6 & 500 & \begin{tabular}{|l|}
0.053 \\
\end{tabular} & 0 & 1.86 & 120.76 & 9.602 & 6 \\
\hline 49 & SMCN & 1 & 560 & \begin{tabular}{|l|}
0.053 \\
\end{tabular} & 0 & 1.999 & 119.952 & 9.063 & 6 \\
\hline 50 & SMCN & 3 & 560 & \begin{tabular}{|l|}
0.053 \\
\end{tabular} & 0 & 2.034 & 100.646 & 9.063 & 6 \\
\hline 51 & BORY & 1 & 500 & \begin{tabular}{|l|}
0.053 \\
\end{tabular} & 0.001 & 1.444 & 222.667 & 8.395 & 6 \\
\hline 52 & BORY & 4 & 500 & \begin{tabular}{|l|}
0.053 \\
\end{tabular} & 0.001 & 1.62 & 161.724 & 8.395 & 6 \\
\hline 53 & HADN & 4 & 500 & \begin{tabular}{|l|}
0.053 \\
\end{tabular} & 0.001 & 1.606 & 167.842 & 8.462 & 6 \\
\hline 54 & BORY & 5 & 500 & \begin{tabular}{|l|}
0.053 \\
\end{tabular} & 0.001 & 1.665 & 162.298 & 8.395 & 6 \\
\hline 55 & HADN & 2 & 500 & \begin{tabular}{|l|}
0.053 \\
\end{tabular} & 0.001 & 1.641 & 160.883 & 8.462 & 6 \\
\hline 56 & HADN & 1 & 500 & \begin{tabular}{|l|}
0.053 \\
\end{tabular} & 0.001 & 1.667 & 156.249 & 8.462 & 6 \\
\hline 57 & SMCN & 2 & 560 & \begin{tabular}{|l|}
0.053 \\
\end{tabular} & 0.001 & 1.642 & 188.31 & 9.063 & 6 \\
\hline 58 & HONM & 1 & 250 & \begin{tabular}{|l|}
0.044 \\
\end{tabular} & 0 & 2.271 & 56.12 & 10.328 & 3 \\
\hline 59 & HONM & 2 & 250 & \begin{tabular}{|l|}
0.044 \\
\end{tabular} & 0 & 2.271 & 56.12 & 10.328 & 3 \\
\hline 60 & YNDN & 12 & 200 & \begin{tabular}{|l|}
0.044 \\
\end{tabular} & 0 & 2.253 & 72.113 & 18.775 & 3 \\
\hline 61 & DNHE & 1 & 200 & \begin{tabular}{|l|}
0.044 \\
\end{tabular} & 0 & 2.238 & 42.252 & 20.965 & 3 \\
\hline 62 & DNHE & 2 & 200 & \begin{tabular}{|l|}
0.044 \\
\end{tabular} & 0.001 & 2.088 & 54.383 & 20.965 & 3 \\
\hline 63 & POSC & 2 & 1000 & \begin{tabular}{|l|}
0.053 \\
\end{tabular} & 0 & 1.534 & 74.532 & 23.999 & 6 \\
\hline 64 & YGWL & 1 & 450 & \begin{tabular}{|l|}
0.053 \\
\end{tabular} & 0.001 & 1.628 & 26.615 & 44.214 & 6 \\
\hline 65 & YGWL & 2 & 450 & \begin{tabular}{|l|}
0.053 \\
\end{tabular} & 0.001 & 1.628 & 26.615 & 44.214 & 6 \\
\hline 66 & WIC1 & 24 & 225 & \begin{tabular}{|l|}
0.026 \\
\end{tabular} & 0 & 1.332 & 77.547 & 41.837 & 3 \\
\hline 67 & ILSN & 22 & 300 & \begin{tabular}{|l|}
0.05 \\
\end{tabular} & 0 & 1.335 & 143.203 & 41.837 & 4 \\
\hline 68 & WIC2 & 27 & 225 & \begin{tabular}{|l|}
0.026 \\
\end{tabular} & 0 & 1.345 & 79.233 & 41.837 & 3 \\
\hline 69 & WIC2 & 26 & 225 & \begin{tabular}{|l|}
0.026 \\
\end{tabular} & 0 & 1.412 & 72.021 & 41.837 & 3 \\
\hline 70 & YNDN & 11 & 125 & \begin{tabular}{|l|}
0.035 \\
\end{tabular} & 0.001 & 2.268 & 40.823 & 24.403 & 3 \\
\hline 71 & WIC2 & 28 & 225 & \begin{tabular}{|l|}
0.026 \\
\end{tabular} & 0.001 & 1.024 & 117.466 & 41.837 & 3 \\
\hline 72 & ICNB & 21 & 504 & \begin{tabular}{|l|}
0.044 \\
\end{tabular} & 0 & 1.56 & 53.548 & 41.837 & 6 \\
\hline 73 & BORB & 23 & 450 & \begin{tabular}{|l|}
0.044 \\
\end{tabular} & 0 & 1.577 & 54.962 & 41.837 & 4 \\
\hline 74 & BUND & \begin{tabular}{|l|}
22 \\
\end{tabular} & 340 & \begin{tabular}{|l|}
0.05 \\
\end{tabular} & 0 & 1.595 & 126.558 & 41.837 & 4 \\
\hline
\end{tabular}

\begin{tabular}{|c|c|c|c|c|c|c|c|c|c|}
\hline 75 & BORB & 24 & 450 & \begin{tabular}{|l|}
0.044 \\
\end{tabular} & 0 & 1.599 & 64.521 & 41.837 & 4 \\
\hline 76 & BORB & 21 & 450 & 0.044 & 0 & 1.608 & 59.26 & 41.837 & 4 \\
\hline 77 & ULSB & 22 & 450 & 0.044 & 0 & 1.609 & $\begin{array}{l}71.325 \\
\end{array}$ & 41.837 & 4 \\
\hline 78 & ULSB & 23 & 450 & 0.044 & 0 & 1.609 & 71.325 & 41.837 & 4 \\
\hline 79 & BUSN & 23 & 450 & 0.044 & 0 & 1.613 & 29.921 & 41.837 & 4 \\
\hline 80 & BUSN & 24 & 450 & 0.044 & 0 & 1.621 & 26.53 & 41.837 & 4 \\
\hline 81 & BUSN & 21 & 450 & 0.044 & 0 & 1.628 & 26.615 & 41.837 & 4 \\
\hline 82 & BUSN & 22 & 450 & 0.044 & 0 & 1.628 & 26.615 & 41.837 & 4 \\
\hline 83 & BORB & 22 & 450 & 0.044 & 0 & 1.631 & 54.619 & 41.837 & 4 \\
\hline 84 & $\begin{array}{l}\text { PYNT } \\
\end{array}$ & \begin{tabular}{|l|}
1 \\
\end{tabular} & 350 & 0.062 & 0 & 1.828 & 101.733 & 37.527 & 4 \\
\hline 85 & SIC1 & 22 & 450 & 0.044 & 0 & 1.643 & 43.561 & 41.837 & 4 \\
\hline 86 & SIC2 & 23 & 450 & 0.044 & 0 & 1.643 & 43.561 & 41.837 & 4 \\
\hline 87 & SIC2 & 24 & 450 & 0.044 & 0 & 1.643 & 43.561 & 41.837 & 4 \\
\hline 88 & WIC1 & 22 & 225 & 0.026 & 0.001 & 1.249 & 86.087 & 41.837 & 3 \\
\hline 89 & $\begin{array}{l}\text { SIC1 } \\
\end{array}$ & 21 & 450 & 0.044 & 0 & 1.662 & $\begin{array}{l}38.765 \\
\end{array}$ & 41.837 & 4 \\
\hline 90 & WIC1 & 23 & 225 & 0.026 & 0.001 & 1.256 & $\begin{array}{l}89.919 \\
\end{array}$ & 41.837 & 3 \\
\hline 91 & $\begin{array}{l}\text { PYNT } \\
\end{array}$ & 2 & 350 & 0.062 & 0 & 1.863 & 97.022 & 37.527 & 4 \\
\hline 92 & $\begin{array}{l}\text { PYNT } \\
\end{array}$ & \begin{tabular}{|l|}
4 \\
\end{tabular} & 350 & 0.062 & 0 & 1.873 & 95.447 & 37.527 & 4 \\
\hline 93 & WIC2 & 25 & 225 & 0.026 & 0.001 & 1.285 & 76.76 & 41.837 & 3 \\
\hline 94 & WIC1 & 21 & 225 & 0.026 & 0.001 & 1.299 & 78.656 & 41.837 & 3 \\
\hline 95 & PYNB & 21 & 480 & 0.044 & 0 & 1.764 & 76.606 & 41.837 & 4 \\
\hline 96 & SECN & 2 & 200 & 0.044 & 0.001 & 2.393 & 52.304 & 28.016 & 3 \\
\hline 97 & SECN & 1 & 200 & 0.044 & 0.002 & 2.12 & 77.257 & 28.016 & 3 \\
\hline 98 & ILSN & 21 & 600 & 0.044 & 0 & 1.798 & 79.812 & 41.837 & 6 \\
\hline 99 & ULSB & 21 & 300 & \begin{tabular}{l|l|}
0.05 \\
\end{tabular} & 0 & 1.853 & 42.16 & 41.837 & 4 \\
\hline 100 & PYNT & \begin{tabular}{|l|l|}
3 \\
\end{tabular} & 350 & 0.062 & 0.001 & 1.662 & 118.091 & 37.527 & 4 \\
\hline 101 & YNAM & 2 & 200 & 0.075 & 0 & 2.227 & 62.344 & 37.521 & 3 \\
\hline 102 & BUND & 21 & 560 & 0.044 & 0 & 2.012 & 63.828 & 41.837 & 6 \\
\hline 103 & SJJU & 4 & 100 & 0.049 & 0.001 & 1.833 & 22.927 & 43.3 & 3 \\
\hline 104 & YESU & \begin{tabular}{|l|}
2 \\
\end{tabular} & 329 & 0.062 & 0.002 & 1.202 & $\begin{array}{l}173.653 \\
\end{array}$ & 39.101 & 4 \\
\hline 105 & WIC1 & \begin{tabular}{|l|}
11 \\
\end{tabular} & 150 & \begin{tabular}{ll|}
0.04 \\
\end{tabular} & 0 & $\begin{array}{l}2.076 \\
\end{array}$ & 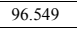 & 41.837 & 3 \\
\hline 106 & WIC1 & 12 & 150 & \begin{tabular}{ll|}
0.04 \\
\end{tabular} & 0 & 2.076 & 96.549 & 41.837 & 3 \\
\hline 107 & WIC1 & 13 & 150 & \begin{tabular}{l|l|}
0.04 \\
\end{tabular} & 0 & 2.076 & 96.549 & 41.837 & 3 \\
\hline 108 & WIC1 & 14 & 150 & \begin{tabular}{ll|}
0.04 \\
\end{tabular} & 0 & 2.076 & 96.549 & 41.837 & 3 \\
\hline 109 & WIC2 & 15 & 150 & \begin{tabular}{l|l|}
0.04 \\
\end{tabular} & 0 & 2.076 & 96.549 & 41.837 & 3 \\
\hline 110 & WIC2 & 16 & 150 & \begin{tabular}{|l|l|}
0.04 \\
\end{tabular} & 0 & 2.076 & 96.549 & 41.837 & 3 \\
\hline 111 & WIC2 & \begin{tabular}{|l|}
17 \\
\end{tabular} & 150 & 0.04 & 0 & 2.076 & 96.549 & 41.837 & 3 \\
\hline 112 & WIC2 & \begin{tabular}{|l|}
18 \\
\end{tabular} & 150 & \begin{tabular}{l|l|}
0.04 \\
\end{tabular} & 0 & 2.076 & 96.549 & 41.837 & 3 \\
\hline 113 & ULSN & \begin{tabular}{|l|}
4 \\
\end{tabular} & 400 & 0.031 & 0.001 & 1.663 & $\begin{array}{l}128.78 \\
\end{array}$ & 38.139 & 4 \\
\hline 114 & ULSN & \begin{tabular}{|l|}
5 \\
\end{tabular} & 400 & 0.031 & 0.001 & 1.663 & $\begin{array}{l}128.78 \\
\end{array}$ & 38.139 & 4 \\
\hline 115 & ULSN & 6 & 400 & 0.031 & 0.001 & 1.663 & $\begin{array}{l}128.78 \\
\end{array}$ & 38.139 & 4 \\
\hline 116 & JEJU & 1 & 40 & 0.031 & 0.025 & 0.364 & 28.484 & 43.599 & 3 \\
\hline 117 & YNAM & $1 \mid$ & 200 & 0.075 & 0.001 & 2.072 & 61.059 & \begin{tabular}{|l|l|}
37.521 \\
\end{tabular} & 3 \\
\hline 118 & ICNC & \begin{tabular}{|l|}
3 \\
\end{tabular} & 325 & 0.05 & 0 & 2.156 & 72.851 & 41.837 & 4 \\
\hline 119 & ICNC & \begin{tabular}{|l|}
4 \\
\end{tabular} & 325 & \begin{tabular}{l|l|}
0.05 \\
\end{tabular} & 0 & 2.173 & 73.848 & 41.837 & 4 \\
\hline 120 & SJJU & \begin{tabular}{|l|l|}
3 \\
\end{tabular} & 100 & $\begin{array}{l}0.049 \\
\end{array}$ & 0.004 & 1.512 & 45.207 & 43.3 & 3 \\
\hline 121 & ICNC & 1 & 250 & 0.026 & 0.001 & 1.823 & 68.429 & 41.837 & 3 \\
\hline 122 & ICNC & 2 & 250 & 0.026 & 0.001 & 1.823 & 68.429 & 41.837 & 3 \\
\hline 123 & NJJU & 3 & 75 & \begin{tabular}{ll|}
0.031 \\
\end{tabular} & 0.001 & 2.016 & 23.401 & 433.599 & 3 \\
\hline 124 & SJJU & \begin{tabular}{|l|} 
\\
\end{tabular} & 20 & 0.031 & 0.006 & 1.999 & 1.36 & 43.3 & 3 \\
\hline 125 & SJJU & 2 & 20 & 0.031 & 0.006 & 1.999 & 1.36 & 43.3 & 3 \\
\hline 126 & YESU & \begin{tabular}{|l|}
1 \\
\end{tabular} & 200 & 0.075 & 0.001 & 2.09 & 43.303 & 39.101 & 3 \\
\hline 127 & NJJU & 2 & 75 & 0.031 & 0.003 & 1.832 & 30.231 & 43.599 & 3 \\
\hline 128 & BUSN & 11 & 300 & 0.05 & 0 & 2.284 & 82.893 & 41.837 & 4 \\
\hline 129 & BUSN & 12 & 300 & 0.05 & 0 & 2.284 & 82.893 & 41.837 & 4 \\
\hline 130 & BUSN & 13 & 300 & \begin{tabular}{l|l|}
0.05 \\
\end{tabular} & 0 & 2.284 & 82.893 & 41.837 & 4 \\
\hline 131 & BUSN & 14 & 300 & \begin{tabular}{l|l|}
0.05 \\
\end{tabular} & 0 & 2.284 & 82.893 & 41.837 & 4 \\
\hline 132 & ULSN & \begin{tabular}{|l|}
1 \\
\end{tabular} & 200 & 0.075 & 0 & 2.228 & 31.473 & 43.207 & 3 \\
\hline 133 & ULSN & \begin{tabular}{|l|l}
3 \\
\end{tabular} & 200 & 0.075 & 0 & 2.228 & 31.473 & 43.207 & 3 \\
\hline 134 & SEOL & 4 & 138 & \begin{tabular}{ll|}
0.04 \\
\end{tabular} & 0.003 & 1.786 & 46.556 & 41.837 & 3 \\
\hline 135 & SEOL & 5 & 250 & 0.026 & 0.002 & 1.633 & 89.488 & 41.837 & 3 \\
\hline 136 & ICNB & 11 & 321 & \begin{tabular}{l|l|}
0.05 \\
\end{tabular} & 0 & $\begin{array}{l}2.389 \\
\end{array}$ & 59.494 & 41.837 & 4 \\
\hline 137 & ULSB & 12 & 300 & \begin{tabular}{l|l|}
0.05 \\
\end{tabular} & 0 & 2.492 & $\begin{array}{l}45.972 \\
\end{array}$ & $\begin{array}{l}41.837 \\
\end{array}$ & 4 \\
\hline 138 & ULSB & 13 & 300 & \begin{tabular}{l|l|}
0.05 \\
\end{tabular} & 0 & 2.492 & $\begin{array}{l}45.972 \\
\end{array}$ & $\begin{array}{l}41.837 \\
\end{array}$ & 4 \\
\hline 139 & SIC1 & 12 & 300 & \begin{tabular}{l|l|}
0.05 \\
\end{tabular} & 0.001 & 2.103 & $\begin{array}{l}91.148 \\
\end{array}$ & $\begin{array}{l}41.837 \\
\end{array}$ & 4 \\
\hline 140 & SIC2 & 13 & 300 & 0.05 & 0.001 & 2.103 & 91.148 & 41.837 & 4 \\
\hline 141 & SIC2 & 14 & 300 & \begin{tabular}{l|l|}
0.05 \\
\end{tabular} & 0.001 & 2.103 & 91.148 & 41.837 & 4 \\
\hline 142 & ULSN & $2 \mid$ & 200 & $\begin{array}{ll}0.075 \\
\end{array}$ & 0.001 & 2.175 & $\begin{array}{l}36.324 \\
\end{array}$ & $\begin{array}{l}43.207 \\
\end{array}$ & 3 \\
\hline 143 & ILSN & 12 & 200 & 0.026 & 0 & 2.576 & 52.137 & 41.837 & 3 \\
\hline 144 & SIC1 & \begin{tabular}{|l|}
11 \\
\end{tabular} & 300 & \begin{tabular}{l|l|}
0.05 \\
\end{tabular} & 0 & 2.688 & 36.055 & 41.837 & 4 \\
\hline 145 & BORB & 13 & 300 & \begin{tabular}{l|l|}
0.05 \\
\end{tabular} & 0.001 & 2.359 & 65.677 & 41.837 & 4 \\
\hline 146 & BORB & 11 & 300 & \begin{tabular}{l|l|}
0.05 \\
\end{tabular} & 0.001 & 2.377 & 62.025 & 41.837 & 4 \\
\hline 147 & BORB & 14 & 300 & \begin{tabular}{|l|l|}
0.05 \\
\end{tabular} & 0.002 & 1.994 & 87.277 & 41.837 & 4 \\
\hline 148 & ULSB & 11 & 200 & 0.026 & 0 & 2.808 & 29.642 & 41.837 & 3 \\
\hline 149 & BORB & 12 & 300 & \begin{tabular}{l|l|}
0.05 \\
\end{tabular} & 0.001 & 2.483 & 57.931 & 41.837 & 4 \\
\hline 150 & ILSN & \begin{tabular}{|l|}
11 \\
\end{tabular} & 400 & 0.044 & 0 & 2.971 & 16.219 & 41.837 & 4 \\
\hline 151 & BUND & 12 & 225 & 0.026 & 0 & 2.997 & 24.846 & 41.837 & 3 \\
\hline 152 & BUND & \begin{tabular}{|l|}
11 \\
\end{tabular} & 375 & \begin{tabular}{l|l|}
0.05 \\
\end{tabular} & 0 & 3.034 & 49.47 & 41.837 & 4 \\
\hline 153 & NJJU & \begin{tabular}{|l|} 
\\
\end{tabular} & 10 & 0.031 & 0.062 & 2.1 & 5.971 & 43.599 & 3 \\
\hline 154 & PYNB & 11 & 320 & \begin{tabular}{l|l|}
0.05 \\
\end{tabular} & 0 & $\begin{array}{l}3.287 \\
\end{array}$ & 9.374 & 41.837 & 4 \\
\hline 155 & HNLM & 21 & 105 & $\begin{array}{l}0.049 \\
\end{array}$ & 0 & 1.948 & 16.8 & 77.909 & 3 \\
\hline 156 & HNLM & 13 & 55 & 0.031 & 0 & 1.944 & 53.78 & 79.905 & 3 \\
\hline 15 & HNLM & 11 & 70 & 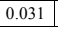 & 0. & 1 & 32 & 9 & \\
\hline
\end{tabular}

Table 2 shows the results obtained the fuzzy set based method for GMS proposed in this paper. In the GMS Table, dotted lines and cross marks mean the weeks of available 
maintenance scheduling and the weeks of maintenance scheduling respectively. It was assumed that generators whose operations start since 2010 need not be included in the maintenance scheduling.

Table 2. Result

\begin{tabular}{|c|c|c|c|c|}
\hline$\ldots \ldots+\ldots+\ldots+\ldots+\ldots+\ldots+\ldots+\ldots+\ldots+\ldots+\ldots+\ldots$ & NG & NM & \begin{tabular}{|l|} 
CAP \\
[MW]
\end{tabular} & \begin{tabular}{|c}
$\begin{array}{c}\text { Init. Poin. } \\
\text { [weeks] }\end{array}$ \\
\end{tabular} \\
\hline-----++++++++---+ & 1 & WLSN & 700 & \\
\hline 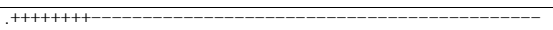 & 2 & WLSN & 679 & 1 \\
\hline 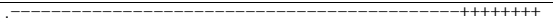 & 3 & WLSN & 700 & 45 \\
\hline .----------------------------------------++++++++-------- & 4 & WLSN & 700 & 37 \\
\hline 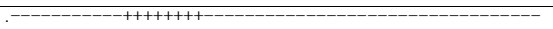 & 5 & ULJN & 950 & 12 \\
\hline .++++++++------------------------------------------------- & 6 & ULJN & 950 & 1 \\
\hline .-----------------------------------------++++++++--------- & 7 & ULJN & 1000 & 37 \\
\hline .--------------------------------------------++++++++ & 8 & ULJN & 1000 & 45 \\
\hline 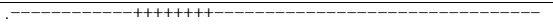 & 9 & YNGN & 950 & 13 \\
\hline 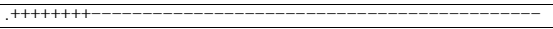 & 10 & YNGN & 950 & 1 \\
\hline .-------------+++++++++--------- & 11 & YNGN & 1000 & 12 \\
\hline 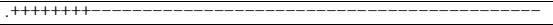 & 12 & YNGN & 1000 & 1 \\
\hline ++++++++ & 13 & NGRI & 1000 & 9 \\
\hline 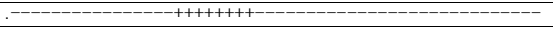 & 14 & GORI & 587 & 17 \\
\hline 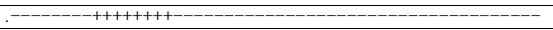 & 15 & GORI & 650 & 9 \\
\hline 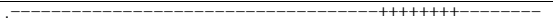 & 16 & GORI & 950 & 37 \\
\hline 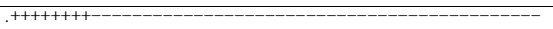 & 17 & GORI & 950 & 1 \\
\hline 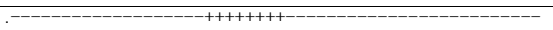 & 18 & ULJN & 1000 & 20 \\
\hline 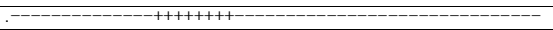 & 19 & YNGN & 1000 & 15 \\
\hline .-------------------------------------------++++++++--------- & 20 & YNGN & 1000 & 37 \\
\hline .---+++++++++---------------------------------------------- & 21 & ULJN & 1000 & 4 \\
\hline 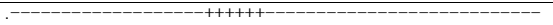 & 22 & HADN & 500 & 20 \\
\hline .--------++++++-------------------------------------- & 23 & BORY & 500 & 9 \\
\hline 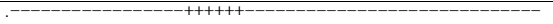 & 24 & DNJN & 500 & 18 \\
\hline $\begin{array}{l}--------+++++++------------------------------------- \\
\end{array}$ & 25 & DNJN & 500 & 9 \\
\hline 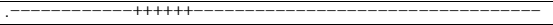 & 26 & HADN & 500 & 13 \\
\hline ---------------------- & 27 & BORY & 500 & 1 \\
\hline & 28 & BORY & 500 & 0 \\
\hline $\begin{array}{l}++++++---------_{-} \\
\end{array}$ & 29 & DNJN & 500 & 1 \\
\hline 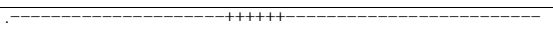 & 30 & DNJN & 500 & 22 \\
\hline 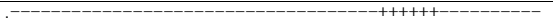 & 31 & DNJN & 500 & 37 \\
\hline 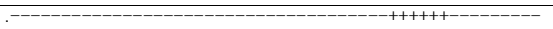 & 32 & DNJN & 500 & 38 \\
\hline 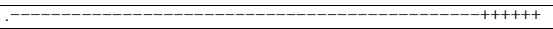 & 33 & DNJN & 500 & 47 \\
\hline .----------------------+++++++--------------------------------- & 34 & DNJN & 500 & 19 \\
\hline .---------------------+++++++--------------------------- & 35 & TEAN & 500 & 21 \\
\hline-----+++++++-- & 36 & TEAN & 500 & 14 \\
\hline 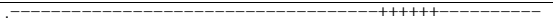 & 37 & TEAN & 500 & 37 \\
\hline.++++++- & 38 & TEAN & 500 & 1 \\
\hline 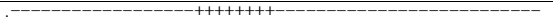 & 39 & YNHN & 800 & 19 \\
\hline-------------++++++- & 40 & SMCN & 500 & 21 \\
\hline--+++++++------ & 41 & SMCN & 500 & 14 \\
\hline------------ & 42 & HADN & 500 & 20 \\
\hline - & 43 & TEAN & 500 & 40 \\
\hline 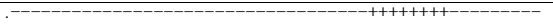 & 44 & YNHN & 800 & 36 \\
\hline 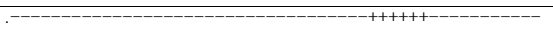 & 45 & SMCN & 560 & 36 \\
\hline 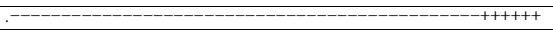 & 46 & TEAN & 500 & 47 \\
\hline 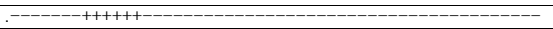 & 47 & TEAN & 500 & 8 \\
\hline 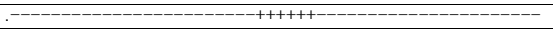 & 48 & TEAN & 500 & 25 \\
\hline .----------------+++++++------------------------------------- & 49 & SMCN & 560 & 15 \\
\hline 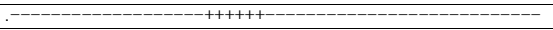 & 50 & SMCN & 560 & 20 \\
\hline 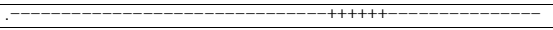 & 51 & BORY & 500 & 32 \\
\hline 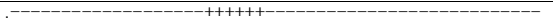 & 52 & BORY & 500 & 20 \\
\hline ---++++++---------------- & 53 & HADN & 500 & 33 \\
\hline----+++++++------ & 54 & BORY & 500 & 41 \\
\hline++++++------ & 55 & HADN & 500 & 9 \\
\hline-----+++++++----- & 56 & HADN & 500 & 16 \\
\hline $\begin{array}{l}++++++---C_{-}-C_{-} \\
\end{array}$ & 57 & SMCN & 560 & 1 \\
\hline 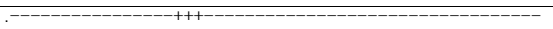 & 58 & HONM & 250 & 17 \\
\hline ---------------+++---------------- & 59 & HONM & 250 & 36 \\
\hline$\overline{----------}$ & 60 & YNDN & 200 & 1 \\
\hline ----------------------------------- & 61 & DNHE & 200 & 1 \\
\hline------ & 62 & DNHE & 200 & 23 \\
\hline 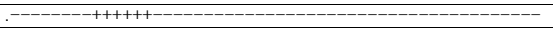 & 63 & POSC & 1000 & 9 \\
\hline++++++- & 64 & YGWL & 450 & 21 \\
\hline------- & 65 & YGWL & 450 & 1 \\
\hline--- & 66 & WICl & 225 & 9 \\
\hline$-\overline{----}$ & 67 & ILSN & 300 & 37 \\
\hline$-\overline{----}$ & 68 & WIC2 & 225 & 23 \\
\hline & 69 & WIC2 & 225 & 1 \\
\hline 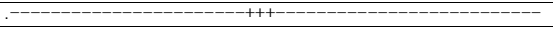 & 70 & YNDN & 125 & 24 \\
\hline$-\overline{-------+++---------------------}$ & 71 & WIC2 & 225 & 31 \\
\hline 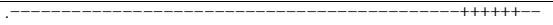 & 72 & ICNB & 504 & 45 \\
\hline---++++- & 73 & BORB & 450 & 17 \\
\hline 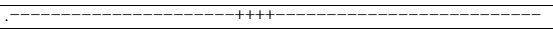 & 74 & BUND & 340 & 23 \\
\hline 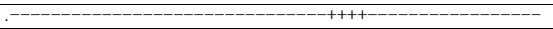 & 75 & BORB & 450 & 32 \\
\hline 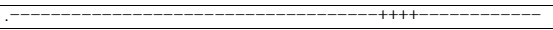 & 76 & BORB & 450 & 37 \\
\hline -------- & 77 & ULSB & 450 & 26 \\
\hline-------- & 78 & ULSB & 450 & 11 \\
\hline.-------++++- & 79 & BUSN & 450 & 7 \\
\hline-+++++------ & 80 & BUSN & 450 & 43 \\
\hline--- & 81 & BUSN & 450 & 32 \\
\hline---++++ & 82 & BUSN & 450 & 49 \\
\hline------------++++- & 83 & BORB & 450 & 26 \\
\hline---------+++++---- & 84 & PYNT & 350 & 45 \\
\hline 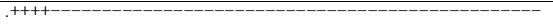 & 85 & SIC1 & 450 & 1 \\
\hline 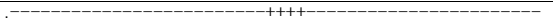 & 86 & SIC2 & 450 & 26 \\
\hline 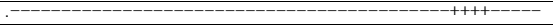 & 87 & SIC2 & \begin{tabular}{|l|l|}
450 \\
\end{tabular} & 44 \\
\hline
\end{tabular}

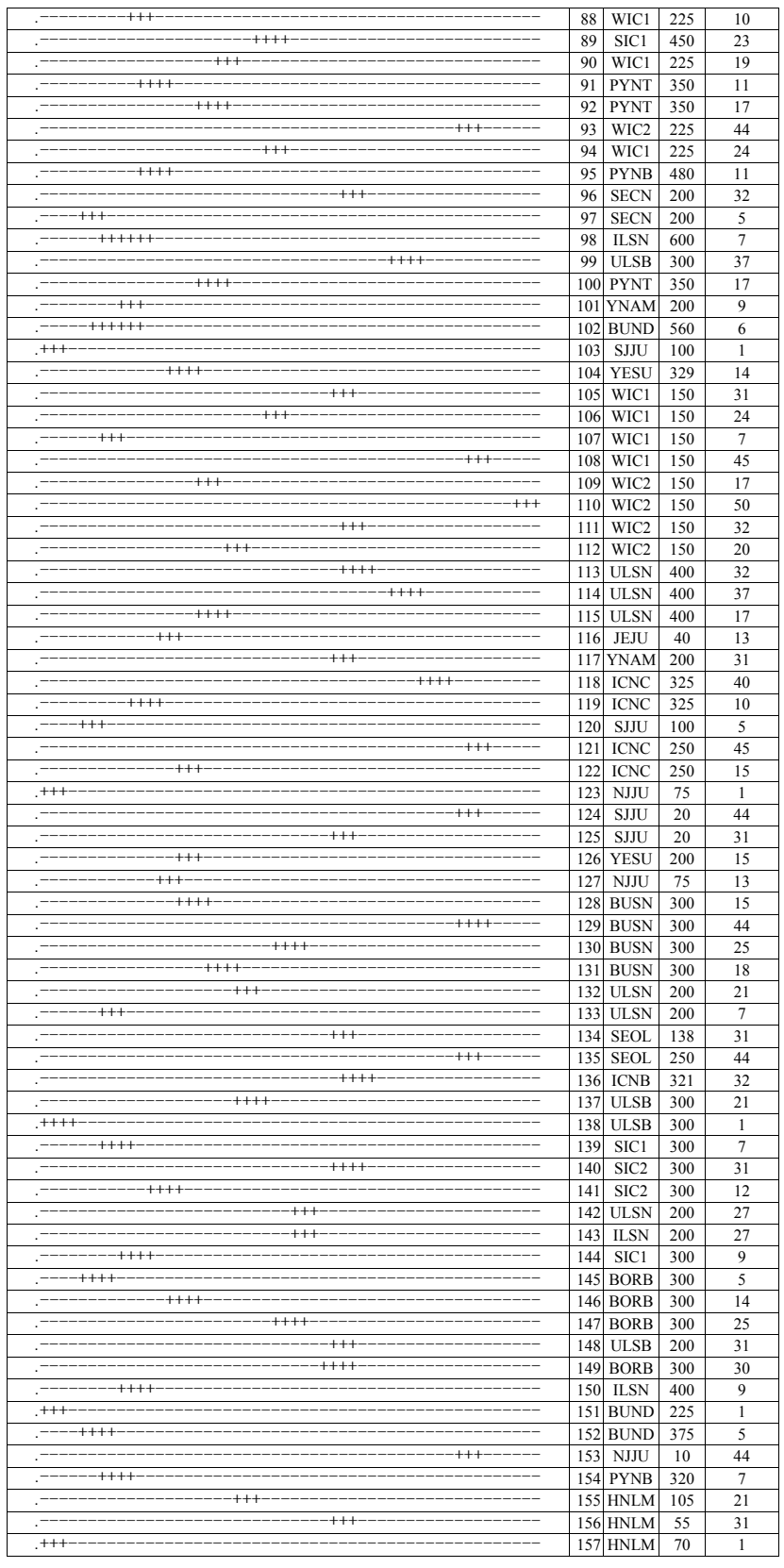

\section{References}

[1] K.Y. Song, Analysis \& operation of Power System, Dongil Publishing Company, 1984.

[2] Roy Billinton, Reliability Assessment of Large Electric Power Systems, Kluwer Academic Publishers, 1986.

[3] X. Wang, J.R. McDonald, Modern Power System Planning, McGraw-Hill Book Company, 1994.

[4] B.Y. Lee, K.B. Shim, "A Comparative Study on Optimal Generation Maintenance Scheduling with Marginal Maintenance Cost and Levelized Risk Method," KIEE, 1992, Vol.41, No.1, pp.9-17.

[5] E.L. Silva, M. Morozowski etc,. "Transmission Constrained Maintenance Scheduling of Generating Units: 
A Stochastic Programming Approach," IEEE Trans. on PS, 1995, Vol.10, No.2, pp.695-701.

[6] M.K.C. Marwali and S.M. Shahidehpour, "Integrated Generation and Transmission Maintenance Scheduling with Network Constraints," IEEE Trans. on PS, 1998, Vol.13, No.3, pp.1063-1068.

[7] J.S. Choi, K.Y. Song, "A Study on Generator Maintenance Scheduling," Proceedings on Summer Meeting of KIEE, 1985, pp.148-151.

[8] J.S. Choi, D.H. Do, T.I. Lee, "Generator Maintenance Scheduling using Fuzzy Multi-criterion Function," Proceedings on Autumn Meeting of KFIS, 1995, pp.131-138.

[9] J.S. Choi, "A Study on the Generator Maintenance Scheduling Considering Load Uncertainty \& MultiCriterion function," EESRI, Final Report, 1995.

[10] J.S. Choi, S.Y. Lee, H.K. Shin, "Generator Maintenance Scheduling using Fuzzy Search Method," Journal of RIACE, Vol.2, 1995, pp.67-73

[11] J.S. Choi, D.H. Do, "Development of a Method for the Generator Maintenance Scheduling using Fuzzy Integer Programming," Journal of KFIS, 1997, Vol. 7, No. 5, pp.77-85.

[12] H.J. Zimmermann, "Fuzzy Set Theory and Its Applications", Kluwer-Nijhohh Boston, 1986, pp.220-234.

[13] Masatoshi Sakawa, "Fuzzy Sets and Interactive Multi-objective Optimization," Plenum Press, 1993, New York.

[14] H.S. Kim, S.P. Moon, D.H. Do, J.S. Choi, S.Y. Lee, H.K. Shin, S.H. Kim, Y.S. Kim, 1998, "Generator Maintenance Scheduling Considering Air Pollution," Proceedings on Autumn Meeting of Western Gyeongnam Area of KIEE, 1998, pp.57-60.

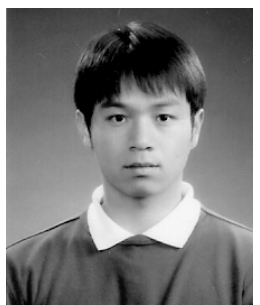

Jeongje Park He received the B.Sc. and M.Sc. degrees from Gyeongsang National University, Jinju, in 2007 and 2008, respectively. His research interest includes probabilistic reliability evaluation of power systems. He is now working forward a Ph.D. degree at Gyeongsang National University.

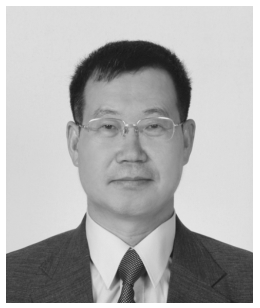

Jaeseok Choi He received the B.Sc., M.Sc., and Ph.D. degrees from Korea University, Seoul, in 1981, 1984, and 1990, respectively. He was a Postdoctoral at the University of Saskatchewan, Saskatoon, SK, Canada, in 1996. Since 1991, he has been on the faculty of Gyeongsang National University, Jinju, Korea, where he is a professor.

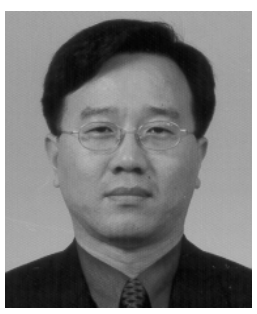

Junmin Cha He obtained B.Sc., M.Sc. and Ph.D. degrees from Korea University in 1989, 1991 and 1996 respectively. His research interest includes Fuzzy Applications, Probabilistic Production Cost Simulation, Reliability Evaluation, Outage Cost Assessment of Power Systems and Operation and Planning of Deregulated Power Market. Since 1996, he has been a faculty of Daejin University, where is now a professor.

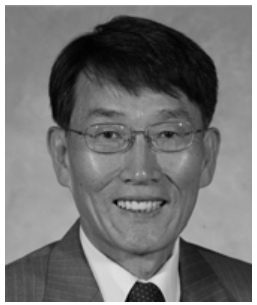

Kwang Y. Lee He received B.Sc. from Seoul National University in Korea, M.Sc. from North Dakota State University and Ph.D. from Michigan State University in 1964, 1968 and 1971 respectively. Since 2007, he has been faculty of Baylor University, where is now a professor. 